\title{
Effect of Remote Ischaemic Preconditioning on Liver Injury in Patients Undergoing Major Hepatectomy for Colorectal Liver Metastasis: A Pilot Randomised Controlled Feasibility Trial
}

\author{
Sanjeev Kanoria ${ }^{1,2}$ - Francis P. Robertson ${ }^{1,2} \cdot$ Naimish N. Mehta ${ }^{1,2}$ • \\ Giuseppe Fusai $^{1,2} \cdot$ Dinesh Sharma $^{1} \cdot$ Brian R. Davidson $^{1,2}$
}

Published online: 8 December 2016

(c) The Author(s) 2016. This article is published with open access at Springerlink.com

\begin{abstract}
Background Liver resection produces excellent long-term survival for patients with colorectal liver metastases but is associated with significant morbidity and mortality from ischaemia reperfusion injury (IRI). Remote ischaemic preconditioning (RIPC) can reduce the effect of IRI. This pilot randomised controlled trial evaluated RIPC in patients undergoing major hepatectomy at the Royal Free Hospital, London.

Methods Sixteen patients were randomised to RIPC or sham control. RIPC was induced through three 10-min cycles of alternate ischaemia and reperfusion to the leg. At baseline and immediately post-resection, transaminases and indocyanine green (ICG) clearance were measured.

Findings The RIPC group had lower ALT and AST levels immediately post-resection (ALT: $43 \%$ lower $497 \pm 165$ vs $889 \pm 170 \mathrm{IU} / \mathrm{L} ; p=0.019$ AST: $54 \%$ lower $408 \pm 166$ vs $836 \pm 167 \mathrm{IU} / \mathrm{L} ; p=0.001$ ) and at $24 \mathrm{~h}$ (ALT: $41 \%$ lower $412 \pm 144$ vs $698 \pm 137 \mathrm{IU} / \mathrm{L} ; p=0.026$ AST: $50 \%$ lower $316 \pm 116$ vs $668 \pm 115 \mathrm{IU} / \mathrm{L} ; p=0.02$ ). ICG clearance was reduced in controls versus RIPC immediately after resection (ICG-PDR: $11.1 \pm 1.1$ vs $16.5 \pm 1.4 \%$ / $\min ; p=0.035$ ).

Conclusions This pilot study shows that RIPC has potential to reduce liver injury following hepatectomy justifying a prospective RCT powered to demonstrate clinical benefits.
\end{abstract}

\section{Introduction}

Liver resection for colorectal metastasis is the gold standard treatment and has improved survival in patients with colorectal liver metastasis [1, 2]. Warm ischaemia reperfusion injury (IRI) to the liver occurs during major liver resections with mobilisation and retraction of the liver and

Francis P. Robertson

francis.robertson.13@ucl.ac.uk

1 Hepato-Pancreatico-Biliary and Liver Transplant Unit, University Department of Surgery, Royal Free Hospital, London NW3 2QG, UK

2 Department of Surgical and Interventional Science, Royal Free Campus, University College London, 9th Floor Royal Free Hospital, Pond Street, London NW3 2QG, UK with the use of temporary portal inflow occlusion (Pringle manoeuvre) [3]. Even in the absence of inflow occlusion, oxygenation of the liver tissue is significantly reduced during mobilisation prior to parenchymal transection [4], resulting in repetitive warm IR injury and significant hepatocyte death prior to parenchymal transection and inflow occlusion [5, 6]. Livers with fibrosis, steatosis or following neo-adjuvant chemotherapy [7] are more susceptible to this warm IR injury. The mean age of patients undergoing liver resection is 60 years [8], and more patients are undergoing neo-adjuvant chemotherapy. As such, strategies to ameliorate IRI are a key clinical concern especially in this group of patients.

Various strategies to reduce IRI to the liver have been described including ischaemic preconditioning (IPC), which may be applied directly or remotely. Ischaemic 
preconditioning reduces the adverse effects of IRI through previous exposure to a brief period(s) of vascular occlusion. Direct IPC has been shown to reduce IRI in experimental models of warm hepatic IRI [9] and in patients undergoing major liver resections [10, 11]. However, experimental studies have shown that direct IPC through clamping of the portal inflow vessels may impair liver regeneration [12, 13] and in a multivariate analysis of patients undergoing liver resection, this was found to be an independent predictor for increased post-operative morbidity [14].

Novel methods of preconditioning without direct stress to the liver include remote ischaemic preconditioning (RIPC) [15]. In RIPC, a remote organ is preconditioned prior to ischaemia of the target organ, and in experimental studies, this has been shown to reduce IRI to the myocardium [16], the liver [17, 18] and other organs [15]. The beneficial effect of RIPC in reducing IRI was first demonstrated in paediatric patients undergoing cardiopulmonary bypass [19]. Results from following clinical trials and meta-analyses in cardiac and vascular surgery have been varied [16, 20-26]. It is unclear why some trials have shown evidence of protection while others have not. This may reflect underlying comorbidities, different conditioning protocols or variability in the potential for a target organ to be preconditioned. As the mechanism by which RIPC provides protection remains unknown, it is difficult to understand what is the reason for these differing results.

We have previously shown that RIPC can reduce liver IRI in an experimental model [17]. This current trial is a proof of concept study to determine whether RIPC reduces IRI in patients undergoing major liver resectional surgery and to help determine potential end points for a subsequent trial to determine the risks and benefits of RIPC in patients undergoing major liver resection for colorectal liver metastases.

In this study, in addition to measuring biochemical parameters of hepatocellular injury, indocyanine green (ICG) clearance from the liver and ICG plasma disappearance rate (ICG-PDR) has been measured which provide reliable early indicators of post-operative liver function [27]. To predict liver injury after hepatectomy and to assess functional hepatic reserve, static measures of liver function such as transaminases [28] have limited reliability. Dynamic tests using ICG clearance and lidocaine metabolism are superior with ICG having the advantage of being measured non-invasively [29]. There is a close correlation between the ICG-PDR and ICG-retention rates measured non-invasively and their corresponding values calculated by conventional ICG methods [30].

\section{Hypothesis}

The hypothesis was that RIPC would be safe and feasible in patients undergoing liver resection surgery and that
RIPC would result in evidence of a reduction in peri-operative liver injury.

\section{Materials and methods}

A single-centre blind prospective randomised controlled trial was performed at the Royal Free Hospital, between April 2005 and April 2007, following approval by the local NHS ethical board $(54,561,358)$. The trial involved randomisation of patients undergoing major liver resection (3 segments or more) for colorectal liver metastasis and was carried out in conjunction with a similar trial in liver transplant recipients. It was registered with ClinicalTrials.gov: Number NCT00796588.

Patients above the age of 18 being considered for major liver resection for colorectal liver metastasis under 3 surgeons were enrolled in the study. Exclusion criteria included: the absence of written informed consent, peripheral vascular disease, blood disorders, e.g. sickle cell disease, localised limb infections, pregnancy, severe comorbid disease, uncontrolled diabetes and sepsis.

Twenty-two patients were assessed for eligibility following which 6 were excluded and 16 were randomised into a control and a RIPC group. For randomisation, computer-generated random numbers were generated and stored in sealed envelopes which were opened following induction of anaesthesia. Patients were blinded to the intervention (RIPC or sham), but the surgeon was not.

\section{The preconditioning stimulus}

In the control group, a sham consisted of a pneumatic tourniquet being placed on the right upper thigh without being inflated. In the RIPC group, following general anaesthesia but before the abdominal incision, the lower limb was covered with two layers of stockinette and elevated to $45^{\circ}$ for $3 \mathrm{~min}$. A wide pneumatic tourniquet was applied to the right upper thigh in accordance with safe and recommended practices by the Association of Peri-operative Registered Nurses (AORN) [31]. To induce RIPC, the tourniquet was inflated to twice the measured systolic arterial pressure for $10 \mathrm{~min}$ and then deflated for $10 \mathrm{~min}$ to reperfuse the leg. This was repeated twice and completed prior to commencing the operation (Fig. 1).

\section{The surgical procedure}

Liver resection was performed through a hockey stick incision. Inflow vessels on the side of the resection were divided extra-parenchymally. Portal vascular inflow occlusion (Pringle manoeuvre) was not required in any of the patients in the study. Liver transection was performed 


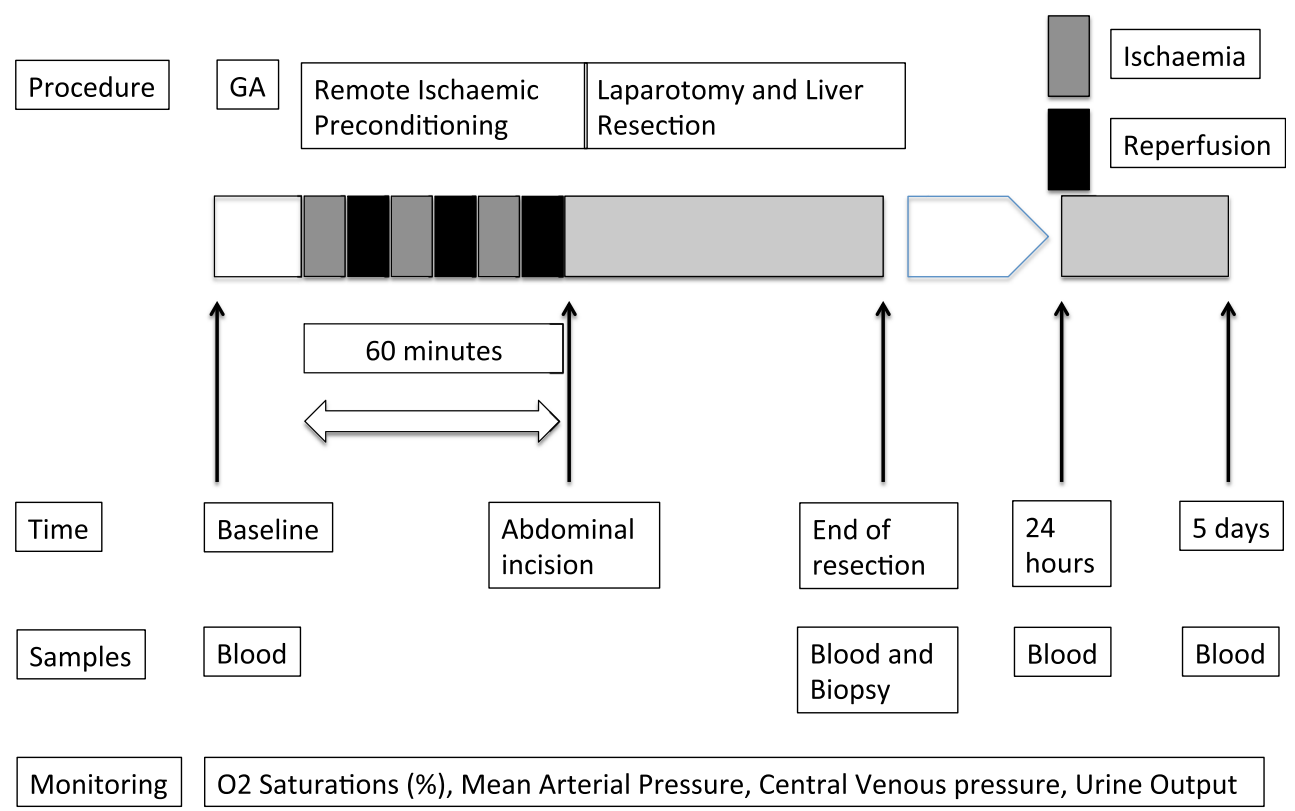

Fig. 1 Trial protocol

using an ultrasonic dissector in all cases. All patients received antibiotic prophylaxis and post-operatively a daily subcutaneous injection of low molecular weight heparin as thrombo-prophylaxis.

\section{Data collection and measurements}

\section{Complications}

During the preconditioning stimulus, patient's haemodynamics were continually monitored. Patients were clinically examined post-operatively for evidence of deep vein thrombosis (DVT) or pulmonary embolus (PE) formation and limb paraesthesia or pain. Doppler ultrasound or CT pulmonary angiogram was requested if there was clinical suspicion of embolus formation.

\section{Blood measurements}

In both groups, $35 \mathrm{mls}$ of peripheral blood was collected at the following time intervals: baseline (following induction of anaesthesia), at the end of the liver resection, and $24 \mathrm{~h}$ post-operatively. Whole blood samples were collected from the arterial line in precooled tubes for the measurement of serum transaminases, serum bilirubin, urea and electrolytes. Measurements were made using an automated clinical chemistry analyser (Hitachi 747, Roche Diagnostics Ltd., Sussex, UK).

\section{Indocyanine green (ICG) pulse densitometry}

ICG is a fluorescent dye eliminated exclusively by the liver, and its elimination rate is used to evaluate global liver function. A bolus of $50 \mathrm{mg}$ ICG (dry powder) [Limon, Pulsion, Munich, Germany] was dissolved in the supplied solvent giving a concentration of $5 \mathrm{mg}$ dye/ml solvent. This was injected slowly intravenously in a dose of $0.5 \mathrm{mg} /$ $\mathrm{kg}$ through the central line. Both absorption and emission spectrum of ICG are in the near-infrared range, and its concentration can be quantified by actual absorbance either invasively with a fibre-optic catheter or non-invasively through the skin [32]. In this trial, the blood concentration of ICG was measured non-invasively via an optical probe attached to the patient's finger and connected to a transcutaneous pulse densitometry monitor (Limon, Pulsion Medical Systems AG, Munich, Germany). Measurements were made at baseline and immediately following completion of liver resection and were recorded as plasma disappearance rate of ICG [ICG-PDR $(\% / \mathrm{min})$ ] and ICG retention rate after $15 \min (R 15 \%)$.

\section{Histological examination}

The resected liver specimen was fixed in $10 \%$ formalin. Tissues were embedded in paraffin and stained with haematoxylin-eosin for histological examination. According to the Royal College of Pathologists guidelines, the resected specimen was evaluated for resection margins, 
nature, distribution and differentiation of the tumour. The normal liver parenchyma was examined for significant signs of IRI, steatosis and fibrosis. Histological evidence of IRI included portal tract inflammation with neutrophil infiltration, hepatocyte ballooning or apoptosis/necrosis and disruption of the trabecular architecture around the central lobar vein. The reporting pathologist was blinded to the trial arm that the patient was allocated to.

\section{Statistical analysis and power calculations}

Although power calculations are not deemed necessary for a pilot feasibility trial, previous studies in direct IPC have achieved a $50 \%$ reduction in serum transaminases in comparison with the control group at $24 \mathrm{~h}$ post-reperfusion $[10,33]$. To demonstrate a benefit of RIPC in reducing liver injury as indicated by a reduction in serum transaminases with a statistical significance $(p<0.05)$, a power of $80 \%$ (two-tailed test of proportions), an $\alpha$-error of 0.05 and a $\beta$-error of 0.00 , it was calculated that a sample size of at least 8 patients per group was required. Distribution of data was analysed by Shapiro-Wilk test and Q-Q plots. Continuous data were expressed as mean $( \pm \mathrm{SD})$, and comparisons between groups were tested by unpaired Student's $t$ test as appropriate. Dichotomous data were presented as a proportion of the whole and comparisons between groups were tested by Chi-squared tests. A $p$ value of $<0.05$ was considered significant, and analysis was by intention to treat.

\section{Results}

Twenty-two patients undergoing liver resection surgery for colorectal liver metastases were approached with 1 patient not wishing to participate in the trial. Five patients were further excluded. Two patients were found to have minor peripheral vascular disease. Three patients who were expected to undergo a major resection underwent a multiple wedge resections and were excluded prior to randomisation. Of the remaining 16 patients, 8 were randomised to the control group and 8 were randomised to the intervention (RIPC) group (Fig. 2). Both groups were well matched at baseline including incidence of pre-operative chemotherapy and degree of steatosis (Table 1).

\section{Safety and feasibility}

Seventy-three percentage of patients approached were recruited to the trial. All patients randomised to the RIPC group successfully underwent RIPC, and there was a $0 \%$ drop out rate post-randomisation.
No patients experienced haemodynamic instability during cuff inflation.

On clinical examination, there was no evidence of DVT or PE formation. No patient required a Doppler USS or CTPA. No patient complained of pain or paraesthesia postoperatively.

\section{Clinical outcomes}

There were no deaths in either group, and no patient suffered from post-operative liver failure. There was a higher incidence of both wound infections ( 2 vs $1, p=0.38$ ) and basal atelectasis ( 4 vs $3, p=0.62$ ) in the control group although neither of these were significant. One patient in the RIPC group developed a post-operative pneumonia, and one patient in the control group developed a postoperative intra-abdominal collection that required radiological drainage. Patients who underwent RIPC spent on average longer in ITU post-operatively although this was not significant ( 2 vs 1.5 days, $p=0.46$ ).

\section{Serum transaminases}

In both groups, serum ALT levels at the end of the resection and at $24 \mathrm{~h}$ post-resection were significantly raised vs baseline (Fig. 3a). At the end of resection, serum ALT levels were $43 \%$ lower in the RIPC group compared to the control group (497 \pm 165 vs $889 \pm 170 \mathrm{IU} / \mathrm{L} ; p=0.019)$. At $24 \mathrm{~h}$ post-resection, ALT levels were $41 \%$ lower in the RIPC group than in the control group $(412 \pm 144$ vs $698 \pm 137 \mathrm{IU} / \mathrm{L} ; p=0.026$ ).

The pattern was similar for the changes in AST. At the end of resection, serum AST levels were 54\% lower in the RIPC group compared with the control group (408 \pm 166 vs $836 \pm 167 \mathrm{IU} / \mathrm{L} ; p=0.001)$. At $24 \mathrm{~h}$ post-resection, serum AST levels were 50\% lower in the RIPC group compared to the control group $(316 \pm 116$ vs $668 \pm 115 \mathrm{IU} / \mathrm{L} ; p=0.02$ ) (Fig. 3b). There were no significant differences in mean serum bilirubin levels between the two groups at the measured time points.

\section{ICG measurements}

The plasma disappearance rate of ICG (ICG-PDR) at baseline in the two groups was similar (control $22.6 \pm 1.9$ vs RIPC $21.5 \pm 1.8 \% / \mathrm{min}$ ). After liver resection, there was a significantly higher ICG clearance in the RIPC group (control $11.1 \pm 1.1 \mathrm{vs} \operatorname{RIPC} 16.5 \pm 1.4 \% / \mathrm{min} ; p=0.035$ ) (Fig. 4a). Similarly the ICG retention at $15 \mathrm{~min}[R 15(\%)]$ at baseline in the two groups was similar (control $6.5 \pm 1.2$ vs RIPC $7.1 \pm 1.6 \%$ ), whereas after liver resection there was a significantly reduced retention of ICG in the RIPC 


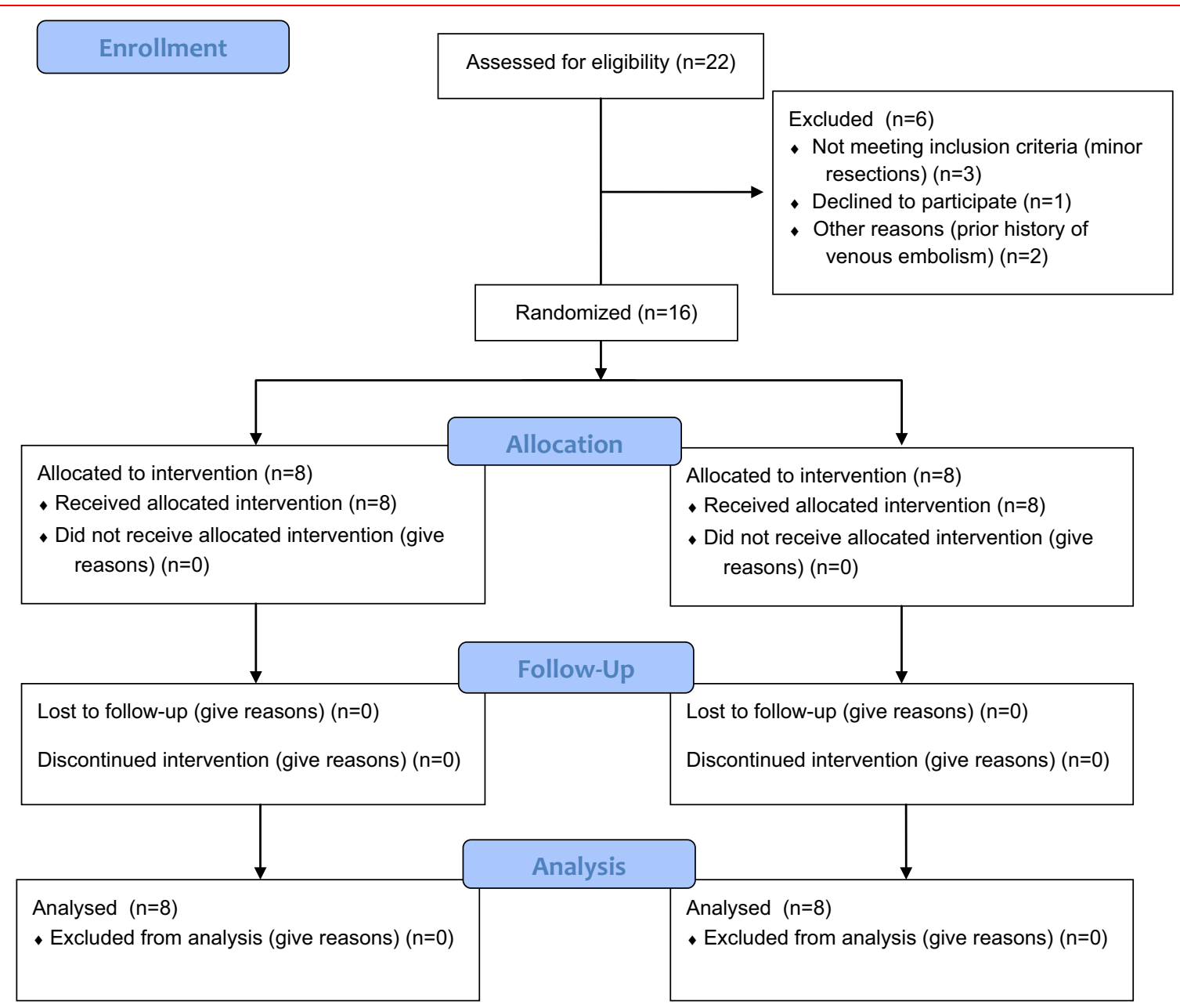

Fig. 2 Randomisation according to the CONSORT guidelines

group (control $17.5 \pm 1.3$ vs $\quad$ RIPC $12.8 \pm 1.6 \%$; $p=0.041$ ) (Fig. 4b).

\section{Histological findings}

In both groups, histology of the liver from the resected specimens was similar to minimal steatosis. There was no pathological evidence of IRI seen.

\section{Discussion}

This pilot feasibility study has shown that RIPC is safe and feasible in patients undergoing liver resection for colorectal liver metastases. Although the trial was not powered to demonstrate improved clinical outcome, a reduction in liver injury as determined by a significant reduction in post-operative serum transaminases and significantly increased ICG clearance was demonstrated in patients undergoing RIPC prior to liver resection.
Liver resection is regarded as the gold standard of treatment for resectable colorectal metastases [1]. The success of the surgery has led to patients requiring extensive parenchymal resections being offered hepatic resection with the risk of post-operative hepatic insufficiency secondary to insufficient residual liver volume. Following surgical resection morbidity can be up to $36 \%$. Besides the extent of the resection, the finding at surgery of liver steatosis and fibrosis is additional important risk factors for post-operative hepatic insufficiency [34] and impaired liver regeneration [35] due to the increased susceptibility to warm IRI [36, 37]. Patients with colorectal metastasis are usually elder (median age 60 years). In experimental models, aged livers have a greater susceptibility to minor degrees of warm IRI [38].

This study has shown that RIPC reduces markers of hepatocellular injury following liver mobilisation in patients undergoing major liver resections and increases the ICG clearance, an important indicator of liver viability [27]. Studies investigating the release of markers of liver parenchymal damage (transaminases and glutathione 
Table 1 Demographic data

\begin{tabular}{lll}
\hline & Control group & RIPC group \\
\hline Age (years) & $66-74$ & $58-77$ \\
Sex ratio (M:F) & $6: 2$ & $7: 1$ \\
BMI (kg/m ${ }^{2}$ & $17-34$ & $22-34$ \\
Operative procedure & & 5 \\
Right hepatectomy & 6 & 2 \\
Extended right hepatectomy & 2 & 1 \\
Left hepatectomy & 0 & 2 \\
Neo-adjuvant chemotherapy & 3 & $6.15(4.7-7.6)$ \\
Duration of operation (h) & $6.25(4.5-8)$ & $9(7-14)$ \\
Intra-operative parameters & & $70(65-80)$ \\
Central venous pressure (mmHg) & $10(8-12)$ & $350(0-750)$ \\
Mean arterial blood pressure $(\mathrm{mmHg})$ & $69(60-77)$ & $300(0-500)$ \\
Blood transfusion (mls) & & \\
\hline
\end{tabular}

Fig. 3 a In both groups, serum

ALT levels at the end of resection and $24 \mathrm{~h}$ postresection were raised versus baseline. At the end of resection, ALT levels were $43 \%$ lower in the RIPC group versus control $(p=0.019)$ and at $24 \mathrm{~h}$ was $41 \%$ lower versus control $(p=0.026)$. b In both groups, serum AST levels at the end of resection and at $24 \mathrm{~h}$ postresection were raised versus baseline. At the end of resection, serum AST levels were $54 \%$ lower in the RIPC group versus control $(p=0.001)$. At $24 \mathrm{~h}$ postresection, serum AST levels were $50 \%$ lower in the RIPC group versus control $(p=0.02)$
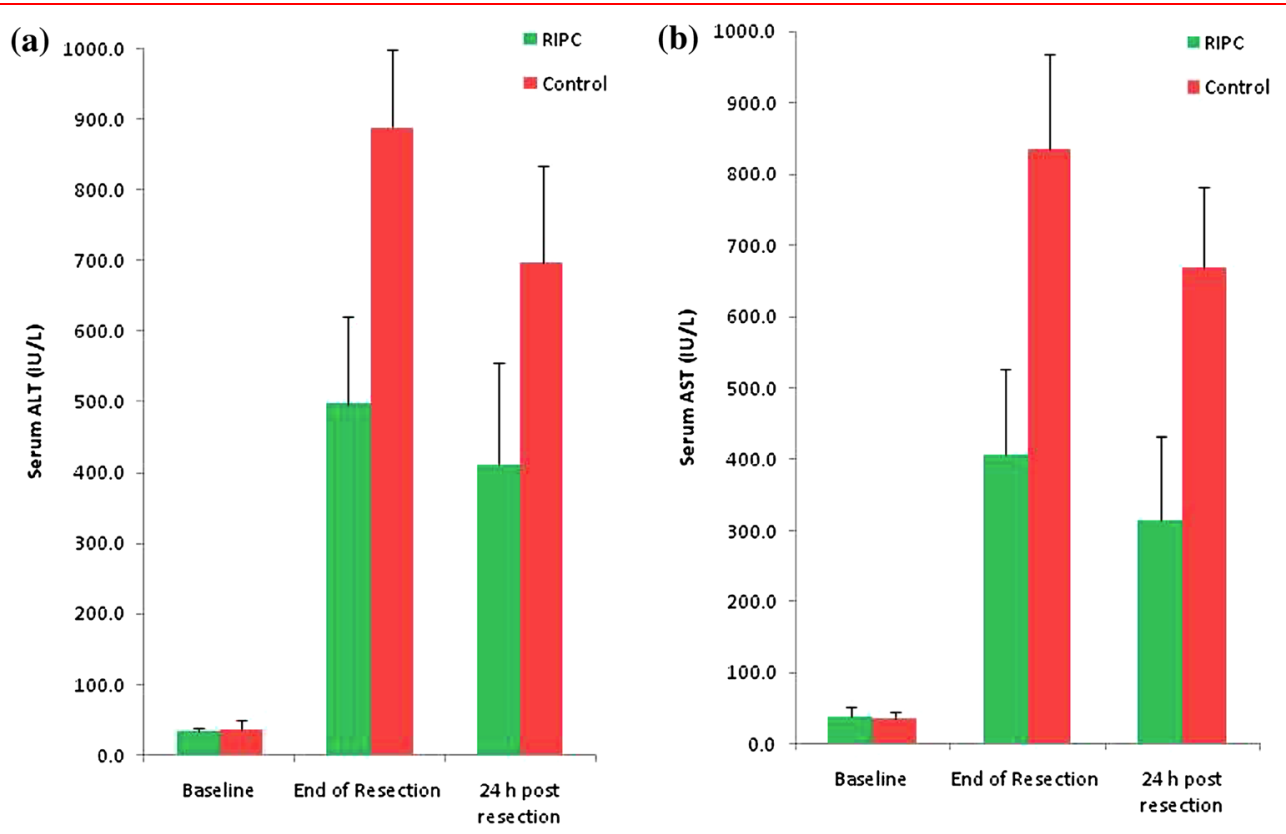

S-transferase) at different stages during and after major liver resection have shown that a significant reduction in hepatic oxygenation occurs during mobilisation of the liver [4] and that markers of parenchymal injury and hepatocyte cell death are significantly raised following mobilisation in the absence of inflow occlusion [5, 6]. This is in keeping with the results from this study which has demonstrated a significant increase in serum transaminase levels in the absence of inflow occlusion and demonstrates that RIPC can protect the liver from this parenchymal injury. This is important as the potential benefits RIPC can be provided more globally rather than only in patients undergoing inflow occlusion.
No patients in this trial had steatosis or fibrosis of the liver; however, 5 patients had undergone neo-adjuvant chemotherapy. If these results can be reproduced in a more extensive trial including patients with risk factors for postoperative liver insufficiency (elderly, prolonged chemotherapy, steatosis, reduced residual liver volume), this may translate into a reduction in the morbidity and mortality associated with liver resection.

The two groups in the trial were well matched for baseline clinical characteristics and indications for hepatectomy. Following major liver resection, serum transaminases increase, peak between 24 and $36 \mathrm{~h}$ and return to normal levels within 3-5 days [39]. The severity of IRI is 
Fig. 4 a ICG-PDR of $<15 \%$ /

min indicates borderline liver

function. ICG-PDR at baseline

in the two groups was not

different, but immediately after

liver resection there was a

significant difference between

the two groups $(p=0.035)$.

b ICG retention of $>15 \%$ after

15 min [ICG-R $15(\%)$ ] after

ICG injection is an indicator of

severe liver dysfunction. ICG-R

$15(\%)$ at baseline in the two

groups were the same, but

immediately after liver resection

there was a significant

difference between the two

groups $(p=0.041)$
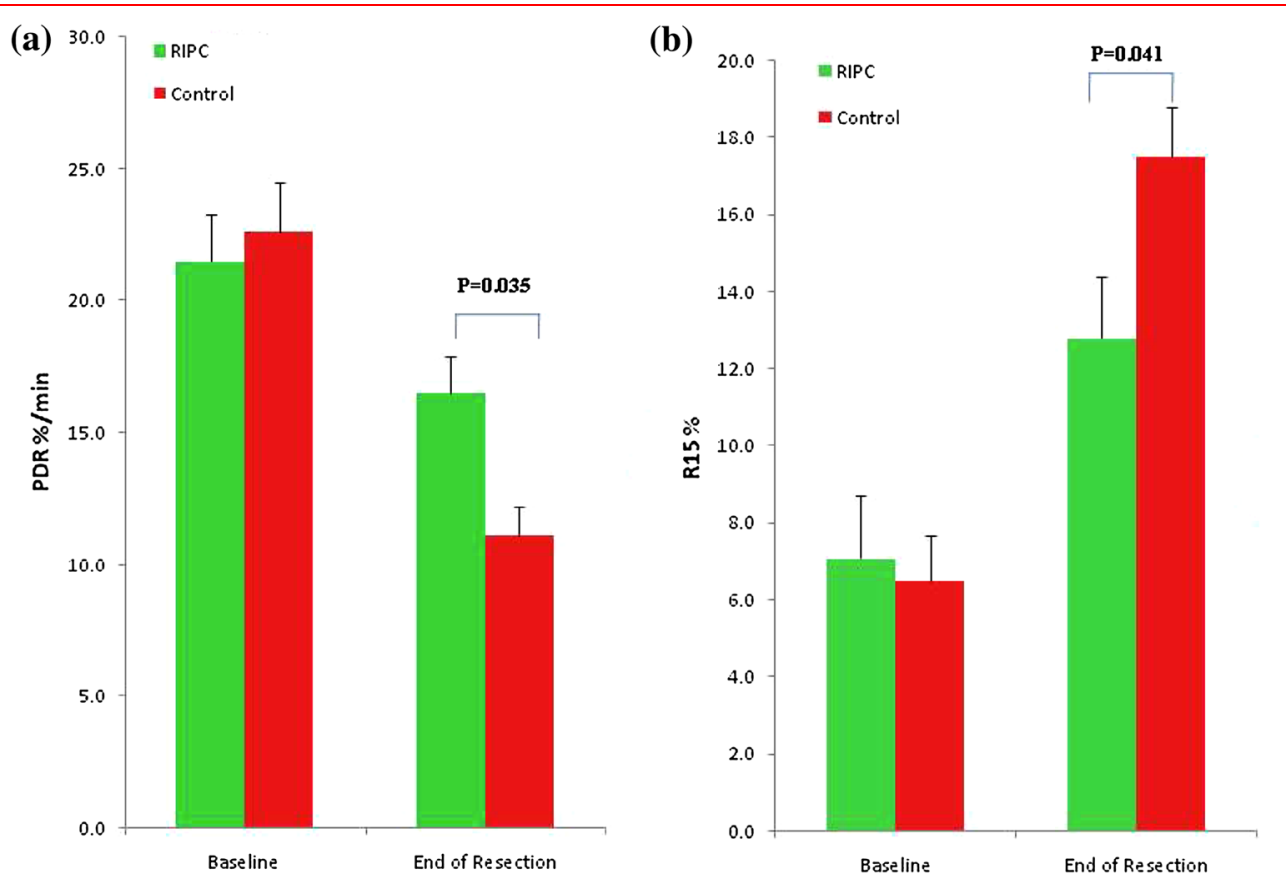

reflected by a rise in serum transaminase levels. In this study in all patients in the two groups, both serum AST and ALT levels increased at the end of the liver resection, peaked at $24 \mathrm{~h}$ and returned to normal in 5 days. RIPC produced a significant reduction in serum transaminases at the end of surgery and at $24 \mathrm{~h}$ post-reperfusion. There was no difference between RIPC and sham groups at 5 days. Direct IPC of the liver in patients undergoing liver resection [10] and liver transplantation [33] also results in a reduction in serum transaminases. However, in experimental studies direct IPC has been shown to impair liver regeneration $[12,13]$ and is associated with a higher risk of post-operative morbidity in patients undergoing major hepatectomy [14]. Hence, in steatotic and small remnant livers even this short duration of direct stress to the liver may be detrimental. This stress can be avoided through remote preconditioning. A limitation of this trial, however, is that RIPC has not been compared against direct IPC of the liver; however, in such a small pilot trial there was insufficient numbers to perform this. Furthermore, the primary aim of this study was to check for feasibility and safety of limb RIPC. Future large RCTs should incorporate a direct IPC arm to allow for a comparison between IPC and RIPC.

Indocyanine green (ICG) is eliminated by the liver largely unchanged into bile and does not undergo enterohepatic recirculation. Elimination is dependent on several factors including hepatic blood flow, hepatocellular uptake and biliary excretion. Hence, the rate of disappearance from the plasma [ICG-PDR $(\% / \mathrm{min})]$ and its percentage retention in the liver at $15 \min [R 15(\%)]$ is a dynamic measure of liver function describing the functional status of the liver at the time of assessment [27]. ICG retention of $>15 \%$ after $15 \mathrm{~min}$ of a bolus injection of ICG is an indicator of significant liver dysfunction and a predictor of reduced patient survival following major liver resections [40]. Measurement of ICG-PDR is more sensitive than serum enzyme tests for assessing liver dysfunction and predicting outcome [41]. In liver transplantation, ICG-PDR measured immediately after liver reperfusion is useful for early diagnosis of primary graft dysfunction and allografts with ICG-PDR of $<15 \% / \mathrm{min}$ have borderline function [42]. Levels below 5\%/min are associated with a high risk of graft failure [27]. In this trial, RIPC improved liver function as demonstrated by an increased ICG plasma clearance and a reduced ICG retention when compared with the control group. Although a reduction in liver injury and improved liver function following RIPC has been identified in this study by reduced transaminases and increased ICG clearance, further sensitive markers of liver injury could be incorporated into future trials including liver fatty acid binding proteins and glutathione S-transferase to further clarify the protection gained by RIPC.

A limitation of this study is that it has not investigated for potential mechanisms of the protection of RIPC. Liver injury propagates an inflammatory response [5], and it has been shown in animal models that mice lacking CD4+ T cells are protected from warm hepatic IRI [43]. Analysis of serum cytokines (IL-6, IFN $\gamma$ and TNF $\alpha$ ) during and postliver resection could be incorporated into future studies to 
measure the inflammatory response and identify the effect of RIPC on this mechanism of injury.

This study has demonstrated the safety of inducing RIPC using a pneumatic limb tourniquet. A standard orthopaedic tourniquet was utilised in the trial as would be used to perform limb surgery in a bloodless field. Tourniquet-associated complications including pain, paraesthesia, pressure changes and haemodynamic disturbances usually occur continuous inflation for more than half an hour [44] and were not observed at any stage during this trial. Standard safety and utilisation guidelines would appear to be adequate to guide the use of a pneumatic tourniquet for producing remote preconditioning.

The benefit of RIPC has not been previously demonstrated in liver resection surgery. Clinical trials in cardiovascular surgery have shown that RIPC may reduce myocardial injury. The benefit of RIPC was first shown in children undergoing cardiac surgery [19]. Subsequently, RIPC was shown to reduce myocardial injury in patients undergoing coronary artery bypass surgery $[16,22]$ and the incidence of peri-operative infarcts in those undergoing aortic surgery [21]. Two large recent trials, however, have failed to demonstrated clinical benefit following RIPC in the setting of cardiac surgery $[25,26]$. What is clear from these trials is that differing protocols for the preconditioning stimulus have been used with varying numbers of cycles (2 vs 4 ) and different methods of vascular occlusion (direct vascular clamping vs limb tourniquet). It has previously been shown in animal models that clamping of the femoral pedicle was as efficacious as a limb tourniquet in ameliorating IR injury [45]. What has not been elucidated is the number and length of cycles that are required to effectively precondition humans. In trials of IPC of donors during liver transplantation, 5 min of portal inflow occlusion was found to be insufficient to reduce IR injury [46] while $10 \mathrm{~min}$ of portal inflow occlusion was shown to reduce post-operative transaminase levels [33]. Three cycles of 5 min have been sufficient to ameliorate IR injury in small animal models [15], but there is no consensus as to what stimulus is required to adequately precondition humans. In this trial, we performed 3 by 10 -min cycles. This longer period of limb ischaemia may explain why RIPC has ameliorated IR injury in this trial compared to others which have used 5-min cycles [25, 26]. It is difficult, however, to draw direct conclusions as the target organ to protect and the limb used (upper vs lower) were different. A further trial comparing 5-min cycles against 10-min cycles is necessary to answer this question.

This clinical study was designed as a pilot feasibility RCT to determine whether patients would be willing to be recruited to a limb preconditioning study with the possibility that they would be randomised to a sham and whether there were risks involved in limb preconditioning using a pneumatic tourniquet. A cohort study using a historical control group would have allowed a comparison between a greater number of patients but would not provide the data on recruitment to help design a future large RCT and would introduce bias due to advances in parenchymal transection techniques and intra-operative blood loss management. Secondary end points which could be used to power a future clinical trial aimed at evaluating the efficacy of RIPC in patients undergoing major liver resections were measured. We have demonstrated that the procedure is acceptable to patients and have found no evidence of complications following RIPC. Surprisingly for a pilot study, we have demonstrated a statistically significant benefit to RIPC in terms of lower post-operative transaminases and improved ICG clearance. These pilot data would justify a prospective clinical trial determining whether RIPC can improve clinical outcomes in major liver surgery.

Acknowledgements We gratefully acknowledge financial support from the Peter Samuel Royal Free Grant to purchase the equipment for measuring indocyanine green clearance, and Prof. Marc Winslet for the support of the academic department of surgery, Royal Free Campus, UCL, and the Wellington Hospital, London, for fellowship support for Mr Francis Robertson.

Funding Funding for this study was provided by Peter Samuel Trust and Wellington Hospital, London.

Open Access This article is distributed under the terms of the Creative Commons Attribution 4.0 International License (http://crea tivecommons.org/licenses/by/4.0/), which permits unrestricted use, distribution, and reproduction in any medium, provided you give appropriate credit to the original author(s) and the source, provide a link to the Creative Commons license, and indicate if changes were made.

\section{References}

1. Adam R et al (2012) The oncosurgery approach to managing liver metastases from colorectal cancer: a multidisciplinary international consensus. Oncologist 17:1225-1239

2. Quan D et al (2012) The role of liver resection for colorectal cancer metastases in an era of multimodality treatment: a systematic review. Surgery 151:860-870

3. Man K et al (1999) Tolerance of the liver to intermittent pringle maneuver in hepatectomy for liver tumors. Arch Surg 134:533-539

4. Kretzschmar M, Krüger A, Schirrmeister W (2003) Hepatic ischemia-reperfusion syndrome after partial liver resection (LR): hepatic venous oxygen saturation, enzyme pattern, reduced and oxidized glutathione, procalcitonin and interleukin-6. Exp Toxicol Pathol 54:423-431

5. van de Poll MCG et al (2007) Liver manipulation causes hepatocyte injury and precedes systemic inflammation in patients undergoing liver resection. World J Surg 31:2033-2038. doi:10. 1007/s00268-007-9182-4

6. Choukér A et al (2005) Alpha-glutathione S-transferase as an early marker of hepatic ischemia/reperfusion injury after liver 
resection. World J Surg 29:528-534. doi:10.1007/s00268-0047431-3

7. Kneuertz PJ, Maithel SK, Staley CA, Kooby DA (2011) Chemotherapy-associated liver injury: impact on surgical management of colorectal cancer liver metastases. Ann Surg Oncol $18: 181-190$

8. Pulitanò $\mathrm{C}$ et al (2010) What defines 'cure' after liver resection for colorectal metastases? Results after 10 years of follow-up. HPB 12:244-249

9. Selzner $N$ et al (2003) Ischemic preconditioning protects the steatotic mouse liver against reperfusion injury: an ATP dependent mechanism. J Hepatol 39:55-61

10. Clavien PA, Yadav S, Sindram D, Bentley RC (2000) Protective effects of ischemic preconditioning for liver resection performed under inflow occlusion in humans. Ann Surg 232:155-162

11. Simillis C, Robertson FP, Afxentiou T, Davidson BR, Gurusamy KS (2015) A network metaanalysis comparing perioperative outcomes of interventions aiming to decrease ischemia reperfusion injury during elective liver resection. Surgery. doi:10.1016/j. surg.2015.10.011

12. Yao A et al (2007) Impaired hepatic regeneration by ischemic preconditioning in a rat model of small-for-size liver transplantation. Transpl Immunol 18:37-43

13. Eipel $\mathrm{C}$ et al (2005) Ischemic preconditioning impairs liver regeneration in extended reduced-size livers. Ann Surg 241:477-484

14. Konopke R et al (2009) Colorectal liver metastasis surgery: analysis of risk factors predicting postoperative complications in relation to the extent of resection. Int J Colorectal Dis 24:687-697

15. Kanoria S, Jalan R, Seifalian AM, Williams R, Davidson BR (2007) Protocols and mechanisms for remote ischemic preconditioning: a novel method for reducing ischemia reperfusion injury. Transplantation 79:445-458

16. Hausenloy DJ et al (2007) Effect of remote ischaemic preconditioning on myocardial injury in patients undergoing coronary artery bypass graft surgery: a randomised controlled trial. Lancet 370:575-579

17. Kanoria $S$ et al (2006) Remote ischaemic preconditioning of the hind limb reduces experimental liver warm ischaemia-reperfusion injury. Br J Surg 93:762-768

18. Lai I-R, Chang K-J, Chen C-F, Tsai H-W (2006) Transient limb ischemia induces remote preconditioning in liver among rats: the protective role of heme oxygenase-1. Transplantation 81:1311-1317

19. Cheung $\mathrm{MMH}$ et al (2006) Randomized controlled trial of the effects of remote ischemic preconditioning on children undergoing cardiac surgery: first clinical application in humans. J Am Coll Cardiol 47:2277-2282

20. Walsh SR, Tang TY, Sadat U, Gaunt ME (2009) Remote ischemic preconditioning in major vascular surgery. J Vasc Surg 49:240-243

21. Ali ZA et al (2007) Remote ischemic preconditioning reduces myocardial and renal injury after elective abdominal aortic aneurysm repair: a randomized controlled trial. Circulation 116:I98-I105

22. Candilio L et al (2015) Effect of remote ischaemic preconditioning on clinical outcomes in patients undergoing cardiac bypass surgery: a randomised controlled clinical trial. Heart 101:185-192

23. Takagi H, Manabe H, Kawai N, Goto S-N, Umemoto T (2008) Review and meta-analysis of randomized controlled clinical trials of remote ischemic preconditioning in cardiovascular surgery. Am J Cardiol 102:1487-1488

24. Healy DA et al (2014) Remote preconditioning and major clinical complications following adult cardiovascular surgery: systematic review and meta-analysis. Int J Cardiol 176:20-31
25. Meybohm P et al (2015) A multicenter trial of remote ischemic preconditioning for heart surgery. N Engl J Med 373:1397-1407

26. Hausenloy DJ et al (2015) Remote ischemic preconditioning and outcomes of cardiac surgery. N Engl J Med 373:1408-1417

27. Kimura $S$ et al (2001) Indocyanine green elimination rate detects hepatocellular dysfunction early in septic shock and correlates with survival. Crit Care Med 29:1159-1163

28. Boleslawski E et al (2014) Relevance of postoperative peak transaminase after elective hepatectomy. Ann Surg. doi:10.1097/ SLA.0000000000000942

29. Sakka SG (2007) Assessing liver function. Curr Opin Crit Care 13:207-214

30. Ishigami $\mathrm{Y}$ et al (1993) Clinical applications of ICG Finger Monitor in patients with liver disease. J Hepatol 19:232-240

31. AORN Recommended Practices Committee (2002) Recommended practices for use of the pneumatic tourniquet. AORN J 75:379-382, 384-386

32. Sakka SG, Reinhart K, Meier-Hellmann A (2000) Comparison of invasive and noninvasive measurements of indocyanine green plasma disappearance rate in critically ill patients with mechanical ventilation and stable hemodynamics. Intensive Care Med 26:1553-1556

33. Jassem $\mathrm{W}$ et al (2006) Ischemic preconditioning of cadaver donor livers protects allografts following transplantation. Transplantation 81:169-174

34. Gomez D et al (2007) Steatosis predicts postoperative morbidity following hepatic resection for colorectal metastasis. Br J Surg 94:1395-1402

35. Veteläinen R, van Vliet A, Gouma DJ, van Gulik TM (2007) Steatosis as a risk factor in liver surgery. Ann Surg 245:20-30

36. Veteläinen R, van Vliet AK, van Gulik TM (2007) Severe steatosis increases hepatocellular injury and impairs liver regeneration in a rat model of partial hepatectomy. Ann Surg 245:44-50

37. Selzner M, Clavien PA (2001) Fatty liver in liver transplantation and surgery. Semin Liver Dis 21:105-113

38. Selzner M, Selzner N, Jochum W, Graf R, Clavien P-A (2007) Increased ischemic injury in old mouse liver: an ATP-dependent mechanism. Liver Transplant 13:382-390

39. Clavien P-A et al (2003) A prospective randomized study in 100 consecutive patients undergoing major liver resection with versus without ischemic preconditioning. Ann Surg 238:843-852

40. Suda K et al (2009) Risk factors of liver dysfunction after extended hepatic resection in biliary tract malignancies. Am J Surg 197:752-758

41. Scheingraber $S$ et al (2008) Indocyanine green disappearance rate is the most useful marker for liver resection. Hepatogastroenterology 55:1394-1399

42. Krenn CG et al (1998) Detection of graft nonfunction after liver transplantation by assessment of indocyanine green kinetics. Anesth Analg 87:34-36

43. Zwacka RM et al (1997) CD4 (+) T-lymphocytes mediate ischemia/reperfusion-induced inflammatory responses in mouse liver. J Clin Investig 100:279-289

44. Valli H, Rosenberg PH, Kyttä J, Nurminen M (1987) Arterial hypertension associated with the use of a tourniquet with either general or regional anaesthesia. Acta Anaesthesiol Scand 31:279-283

45. Abu-Amara M et al (2011) Effect of remote ischemic preconditioning on liver ischemia/reperfusion injury using a new mouse model. Liver Transplant 17:70-82

46. Koneru B et al (2005) Ischemic preconditioning in deceased donor liver transplantation: a prospective randomized clinical trial of safety and efficacy. Liver Transplant 11:196-202 